\title{
Quantum Zeno Suppression of Intramolecular Forces
}

\author{
S. Wüster \\ Max Planck Institute for the Physics of Complex Systems, Nöthnitzer Strasse 38, 01187 Dresden, Germany, \\ Department of Physics, Bilkent University, 06800 Çankaya, Ankara, Turkey \\ and Department of Physics, Indian Institute of Science Education and Research, Bhopal, Madhya Pradesh 462 023, India \\ (Received 23 November 2016; revised manuscript received 19 April 2017; published 6 July 2017)
}

\begin{abstract}
We show that Born-Oppenheimer surfaces can intrinsically decohere, implying loss of coherence among constituent electronic basis states. We consider the example of interatomic forces due to resonant dipoledipole interactions within a dimer of highly excited Rydberg atoms, embedded in an ultracold gas. These forces rely on a coherent superposition of two-atom electronic states, which is destroyed by continuous monitoring of the dimer state through a detection scheme utilizing the background gas atoms. We show that this intrinsic decoherence of the molecular energy surface can gradually deteriorate a repulsive dimer state, causing a mixing of attractive and repulsive character. For sufficiently strong decoherence, a Zeno-like effect causes a complete cessation of interatomic forces. We finally show how short decohering pulses can controllably redistribute population between the different molecular energy surfaces.
\end{abstract}

DOI: 10.1103/PhysRevLett.119.013001

Introduction.-Through the extreme properties of Rydberg states [1], ultracold Rydberg physics allows the study of chemical phenomena in otherwise inaccessible regimes. Examples are homonuclear molecules bound over enormous distances and possessing a permanent dipole moment [2-10], control of chemical reactions [11], and nonadiabatic "nuclear" dynamics involving conical intersections $[12,13]$ over vastly inflated length scales [14-16]. In all the latter cases, the role of nuclei plus inner shell electrons in a usual molecule is taken by entire atoms including their valence electron, but the motion of these atoms is also governed by Born-Oppenheimer (BO) surfaces. The latter arise here through resonant electronic dipole-dipole interactions [1], which give large characteristic length scales for these surfaces $\sim \mathcal{O}[10 \mu \mathrm{m}]$.

Since the electrons forming a molecular orbit in Rydberg chemistry are thus many orders of magnitude farther apart than in usual molecules, we enter a previously inaccessible regime where electrons can be strongly coupled to separate perturbing environments. This enables novel physics through the loss of coherence between electronic basis states that constitute a BO surface, thus instrinsic to the energy surface. Previous studies of decoherence in molecules dealt instead with the loss of coherence between vibrational states on a given surface [17-19] or between different BO surfaces [20]. In these cases decoherence can be caused by additional molecular degrees of freedom.

A natural candidate for a quite different perturbing environment in Rydberg chemistry is the cold background gas in which Rydberg molecules or aggregates are typically created. Exploiting the gas will additionally allow dynamical control of decoherence channels [21], in contrast to the typical case in molecular physics.

Here we thus consider a dimer of two dipole-dipole interacting Rydberg atoms, immersed in a background gas of ground-state atoms. The dimer constituents are strongly coupled even when their separation $r$ vastly exceeds the mean ground-state atom spacing $d$, as sketched in Fig. 1. Rydberg excitations within a background gas have been realized, keeping many of the properties of Rydberg states preserved despite the immersion [9,22-30]. Importantly, the background gas offers means to probe the embedded Rydberg system [25,31-34] and to engineer controllable decoherence $[21,35,36]$.

The molecular states governing the dimer are simple coherent superpositions $\left|\varphi_{\text {rep }}\right\rangle=(|p s\rangle+|s p\rangle) / \sqrt{2},\left|\varphi_{\text {att }}\right\rangle=$ $(|p s\rangle-|s p\rangle) / \sqrt{2}$, involving two electronic Rydberg states $|s\rangle$ and $|p\rangle$ of the constituent atoms. The subscripts indicate the repulsive or attractive character of the corresponding BO surfaces. These states are reminiscent of molecular valence bond states such as $\left|\varphi_{g / u}\right\rangle=\left(\left|\phi_{s}(A)\right\rangle\left|\phi_{p}(B)\right\rangle \pm\right.$ $\left.\left|\phi_{p}(A)\right\rangle\left|\phi_{s}(B)\right\rangle\right) / \sqrt{2}$, where $\left|\phi_{x}(A)\right\rangle$ denotes an electronic state $x$ centered on nucleus $A$ [37].

The background gas can now be used to infer the location (motion) and electronic state of these two Rydberg atoms as discussed in Ref. [21]. This allows one to distinguish the two constituents of the superposition $|p s\rangle$ and $|s p\rangle$. We show that the resulting measurement induced decoherence disrupts the superpositions on which the BO surfaces rely, changing the character of forces from purely repulsive to a mixture of attractive and repulsive. Earlier studies on decoherence of dipole-dipole interaction in Rydberg gases did not consider the effect on atomic motion, nor could a dipole-dipole interacting system and a decohering environment be distinguished [36,39-41].

We further show that dipole-dipole acceleration in the dimer can be brought to a complete arrest, furnishing a quantum Zeno effect [42,43] for motional dynamics with experimentally accessible parameters. Finally, we 
demonstrate how short pulses of strong environment coupling (decoherence) can be exploited to shuffle population between BO surfaces, in a further application of quantum state engineering through decoherence [35,44-46].

Scheme and model.-Consider a Rydberg dimer with interatomic separation $r$ embedded in a cold atom cloud of $M$ background atoms, as sketched in Fig. 1. For the compound dimer, we allow only two electronic pair states $\left|\pi_{1}\right\rangle \equiv|p s\rangle$, with the first atom in $|p\rangle=|\nu p\rangle$ and the second in $|s\rangle=$ $|\nu s\rangle$, and the reverse $\left|\pi_{2}\right\rangle \equiv|s p\rangle$. Here $\nu$ is the principal quantum number and angular momentum $l=0,1$ is denoted by $s, p$. The background atoms are initially prepared in the electronic ground state $|g\rangle$ with random positions.

We can introduce dephasing between $\left|\pi_{1,2}\right\rangle$ through imaging the background atoms in a manner sensitive to the state of a nearby dimer atom. To this end, only background atoms are coupled to two laser fields. One, with Rabi frequency $\Omega_{p}$ and detuning $\Delta_{p}$, drives transitions from $|g\rangle$ to a short-lived intermediate state $|e\rangle$. Another laser couples further from $|e\rangle$ to a third level, a Rydberg state $|f\rangle=\left|\nu^{\prime} s\right\rangle, \nu \neq \nu^{\prime}$, with Rabi frequency $\Omega_{c}$ and detuning $\Delta_{c}$. The state $|e\rangle$ spontaneously decays with rate $\Gamma_{p}$ to $|g\rangle$. Overall we realize electromagnetically induced transparency (EIT) in the ladder configuration [47-54].

We have shown in Ref. [21] based on Refs. [32,33] how interactions between the dimer atoms and background atoms in state $|f\rangle$ allow one to infer which dimer atom is in the $|p\rangle$ state, thereby also providing controllable decoherence in the electronic state space spanned by $\left|\pi_{1}\right\rangle$, $\left|\pi_{2}\right\rangle$. In that work, all atomic motion could be ignored in a frozen gas regime. In contrast, we focus here explicitly on the effect of decoherence on the dipole-dipole induced relative motion of the Rydberg dimer. The Hamiltonian for only the dimer is

$\hat{H}=-\frac{\hbar^{2} \nabla_{r}^{2}}{m}+\hat{H}_{d d}, \quad \hat{H}_{d d}=W_{12}(r)\left|\pi_{1}\right\rangle\left\langle\pi_{2}\right|+$ c.c.,

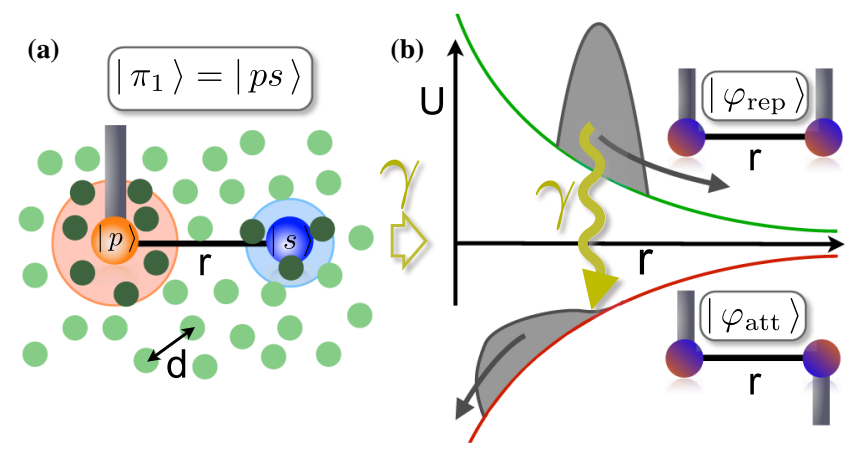

FIG. 1. (a) Sketch of embedded Rydberg dimer. The example shows how the amplitude of $|p\rangle$ excitation (gray bar) in $\left|\pi_{1}\right\rangle$ can be inferred from optical signals within radii $R_{c, s / p}$ (large circles). (b) The dimer atoms [orange and blue in (a)] are initially on a repulsive BO surface $U_{\text {rep }}(r)$ (green line, state $\left|\varphi_{\text {rep }}\right\rangle$ ). Because of decoherence at rate $\gamma$ caused by the background atomic cloud [green in (a)], the population incoherently seeps onto the attractive surface $U_{\text {att }}(r)$ (red line). describing two atoms of mass $m$ with relative coordinate $r$ and dipole-dipole interactions $W_{12}(r)=\mu^{2} / r^{3}\left(W_{n n} \equiv 0\right)$, where $\mu$ is a radial dipole matrix element [55]. Anticipating the decohering effect of the background atoms, we model our system in terms of a density matrix $\hat{\rho}=\sum_{n, m} \int d r d r^{\prime} \rho\left(r, r^{\prime}\right)_{n m}|r\rangle \otimes\left|\pi_{n}\right\rangle\left\langle r^{\prime}\right| \otimes\left\langle\pi_{m}\right|$, the elements of which follow the von-Neumann equation

$$
\begin{aligned}
\dot{\rho}\left(r, r^{\prime}\right)_{\mathrm{nm}}= & -\frac{i}{\hbar}\left[-\frac{\hbar^{2}}{2 m}\left(\nabla_{r}^{2}-\nabla_{r^{\prime}}^{2}\right) \rho\left(r, r^{\prime}\right)_{\mathrm{nm}}\right. \\
& \left.+\sum_{k}\left(W_{n k}(r) \rho\left(r, r^{\prime}\right)_{\mathrm{km}}-W_{\mathrm{km}}\left(r^{\prime}\right) \rho\left(r, r^{\prime}\right)_{\mathrm{nk}}\right)\right] \\
& +\left(i \frac{\Delta E}{\hbar}-\frac{\gamma}{2}\right)\left(1-\delta_{n m}\right) \rho\left(r, r^{\prime}\right)_{\mathrm{nm}} .
\end{aligned}
$$

A derivation of Eq. (2) is given in the Supplemental Material [56], where we also formally define the "position eigenstates" $|r\rangle$ used in the definition of $\hat{\rho}$. The last row of Eq. (2) contains disorder (an energy shift) $\Delta E$ and dephasing $\gamma$ in the dimer electronic state space. Both are tunable through the atomic and optical parameters $\Omega_{p / c}$, $\Delta_{p / c}, \Gamma_{p}$ and interactions of dimer atoms in $|s / p\rangle$ with background gas atoms in $|f\rangle$ as described in Ref. [56].

The dephasing $\gamma$ arises because state-dependent light absorption by the background gas allows one to experimentally distinguish the aggregate states $\left|\pi_{1,2}\right\rangle$. Briefly, interactions cause a breakdown of EIT within a critical radius $R_{c, s / p}$ around a Rydberg atom in $s / p$ as shown in Fig. 1, causing absorption shadows of the corresponding size. When $R_{c, s} \neq R_{c, p}$, observation of the shadow sizes allows discrimination of $\left|\pi_{1,2}\right\rangle$. The shadow centers correspond to the locations of the dimer atoms, so the same mechanism allows the observation of dimer separation $r$.

Since the Rabi frequencies $\Omega_{p / c}(t)$ can vary in time, also $\gamma$ can vary in time; hence, we will refer to it as controllable decoherence. Disorder $\Delta E$ originates from possibly different local environments of background atoms around each aggregate atom.

In the following, we explicitly consider atomic states $|s\rangle=\left|43 s_{\frac{11}{22}}\right\rangle,|p\rangle=\left|43 p_{\frac{31}{22}}\right\rangle$, and $|f\rangle=\left|38 s_{\frac{11}{22}}\right\rangle$ for ${ }^{87} \mathrm{Rb}$, where subscripts indicate angular momentum quantum numbers $\left(j, m_{j}\right)$. See Ref. [15] for a justification of the restriction to these $\left(j, m_{j}\right)$, which is assumed here for simplicity only. For most parameters used, the disorder $\Delta E$ will be negligible and is, hence, set to zero.

Decohering dipole-dipole interactions.-The dipoledipole interaction Hamiltonian $\hat{H}_{d d}$ has two eigenstates $\left|\varphi_{\text {rep } / \text { att }}\right\rangle=\left(\left|\pi_{1}\right\rangle \pm\left|\pi_{2}\right\rangle\right) / \sqrt{2}$, with repulsive and attractive potentials $U(r)_{\text {rep } / \text { att }}= \pm \mu^{2} / r^{3}$ [57-59]. We now investigate the effect of decoherence $\gamma$ on a dimer initialized in the repulsive state

$$
\hat{\rho}(t=0)=\left|\phi_{0}\right\rangle\left|\varphi_{\text {rep }}\right\rangle\left\langle\phi_{0}\right|\left\langle\varphi_{\text {rep }}\right|
$$




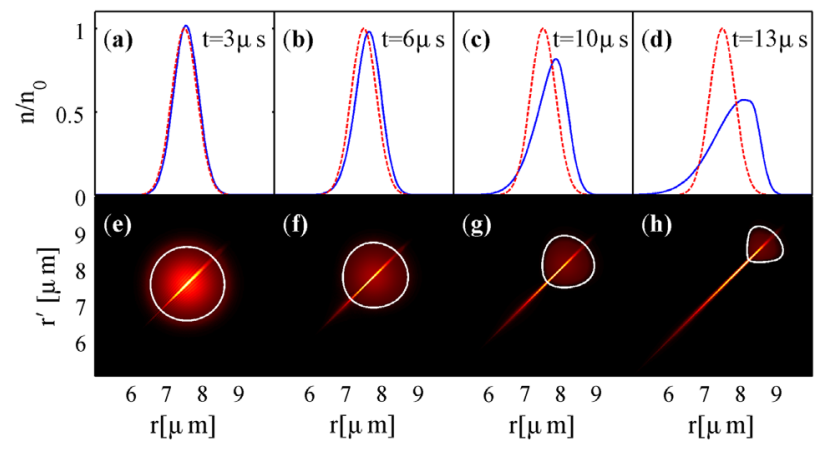

FIG. 2. Continuous decoherence turns an initially repulsive dipole-dipole interaction gradually into a mixture of attractive and repulsive dynamics, according to Eq. (2) for $\gamma=0.2 \mathrm{MHz}$, $r_{0}=7.5 \mu \mathrm{m}, \sigma=0.5 \mu \mathrm{m}$. (a)-(d) Probability density $n(r)=$ $\sum_{k} \rho(r, r)_{\mathrm{kk}}$ of the relative coordinate at the indicated time (blue) and initial time, $t=0$ (red-dashed line), normalized by the initial peak value $n_{0}$. (e)-(h) Corresponding density matrix $\rho\left(r, r^{\prime}\right)_{11}$ at the same times as the upper panels. White lines show the corresponding shape for $\gamma=0$ of only the repulsively evolving fraction [62].

through numerical solutions of Eq. (2) [60,61], as shown in Fig. 2. In the expression above $\left\langle r \mid \phi_{0}\right\rangle=\phi_{0}(r)=$ $\mathcal{N} \exp \left[-\left(r-r_{0}\right)^{2} /\left(2 \sigma^{2}\right)\right]$ represents the initial wave function for the relative coordinate $r$, normalized to $1=$ $\int d r\left|\phi_{0}(r)\right|^{2}$ via $\mathcal{N}$. Thus, $\rho\left(r, r^{\prime}\right)$ is a 2D Gaussian at $t=0$. For short times $t \lesssim 8 \mu$ s we see repulsive acceleration as expected. However, the dephasing terms in Eq. (2) are gradually destroying the quantum coherence between $\left|\pi_{1}\right\rangle$ and $\left|\pi_{2}\right\rangle$, evolving the dimer state towards the incoherent mixed state $\hat{\rho}_{M}=\left[\left|\pi_{1}\right\rangle\left\langle\pi_{1}|+| \pi_{2}\right\rangle\left\langle\pi_{2}\right|\right] / 2$. The latter can also be written as $\hat{\rho}=\left[\left|\varphi_{\text {rep }}\right\rangle\left\langle\varphi_{\text {rep }}|+| \varphi_{\text {att }}\right\rangle\left\langle\varphi_{\text {att }}\right|\right] / 2$; thus, this process will gradually populate the attractive potential. This can be seen in Fig. 2 at later times, where dimer atoms now experience attraction with some probability. It appears that the repulsively moving part of the system (within white contours) has largely preserved the initial spatial phase coherence $\left[\rho\left(r, r^{\prime}\right)>0\right.$ for $\left.\left|r-r^{\prime}\right| \lesssim \sigma\right]$, while the attractively evolving part of the system has lost its phase coherence with the remainder, as it was created through an incoherent process. This view is corroborated by an analysis using a basis change $\left\{\left|\pi_{1,2}\right\rangle\right\} \rightarrow\left\{\left|\varphi_{\text {rep/att }}\right\rangle\right\}$.

Zeno arrest of motion.-For decoherence rates $\gamma$ small compared to the initial dipole-dipole interaction $W_{12}\left(r_{0}\right) \approx 2.2 \mathrm{MHz}$, the slow decoherence studied above essentially acts as a transfer channel onto the attractive potential surface. For much larger decoherence rates, we observe a total arrest of the acceleration of the dimer through dipole-dipole interactions as shown in Fig. 3. For these large $\gamma$, the coherences between $\left|\pi_{1}\right\rangle$ and $\left|\pi_{2}\right\rangle$ are quickly damped close to zero following switch on of controllable decoherence. This is seen in the off-diagonal matrix element $\rho_{21}^{(\text {el })}$ of the reduced electronic density matrix $\hat{\rho}^{(\mathrm{el})}=\operatorname{Tr}_{r}[\hat{\rho}]$ shown in Fig. 3(a) [63]. Dephasing subsequently forces them
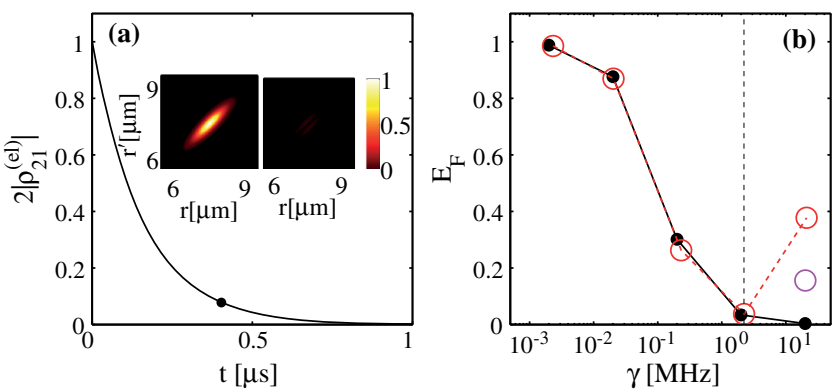

FIG. 3. (a) Rapid loss of coherence between $\left|\pi_{1}\right\rangle$ and $\left|\pi_{2}\right\rangle$ after initiating controllable decoherence with $\gamma=2 \mathrm{MHz}$. The left [right] inset shows the spatial coherence $\rho\left(r, r^{\prime}\right)_{11}\left[\rho\left(r, r^{\prime}\right)_{12}\right]$ at the time indicated by $(\bullet)$. (b) Final kinetic energy of the dimer $E_{f}$, relative to the coherent case as discussed in the text, as a function of decoherence rate $\gamma$. The dashed vertical line indicates $W_{12}\left(r_{0}\right)$. Parameters other than $\gamma$ are as in Fig. 2. The red-dashed line and markers show results using a model including disorder for atom density $n_{0}=5 \times 10^{17} \mathrm{~m}^{-3}$, discussed in Ref. [56]. (magenta circle, $n_{0}=5 \times 10^{18} \mathrm{~m}^{-3}$ ).

to remain near zero, while being opposed in this by dipoledipole interactions. For $\rho\left(r, r^{\prime}\right)_{12}=\rho\left(r, r^{\prime}\right)_{21} \approx 0$ one can see from Eq. (2) that little acceleration will take place, since all terms $\sim W_{12}(r)$ are very small. This reflects that dipoledipole forces essentially rely on the coherence between the two basis states $\left|\pi_{1}\right\rangle$ and $\left|\pi_{2}\right\rangle$; see the expression for the potential energy derived in Ref. [56]. To quantify the arrest of acceleration, we show in Fig. 3(b) the normalized final kinetic energy $E_{f}=\left[E_{\text {kin }}\left(t_{f}\right)-E_{\text {kin }}(0)\right] / E_{0}[56,64]$ of the dimer. At the chosen final time, $t_{f}=19.8 \mu \mathrm{s}$, the dimer has been significantly accelerated to kinetic energy $E_{\text {kin }}\left(t_{f}\right)-$ $E_{\text {kin }}(0)=E_{0}$ in the fully coherent case with $\gamma=0$, for which, hence, $E_{f}=1$. For rates $\gamma$ around $W_{12}\left(r_{0}\right) \approx$ 2.2 MHz, which are in reach of experiments [56], acceleration is almost entirely suppressed.

We recognize a quantum Zeno effect, in which a system that is sufficiently frequently measured is frozen in the quantum state attained after collapse of the wave function $[42,43]$. Since the dephasing rate $\gamma$ arises through gathering position and state information about our embedded Rydberg dimer, the results in Fig. 3 furnish a position space quantum Zeno effect, intriguingly close in spirit to the original philosophy of Zeno regarding the motion of an arrow.

The figure also includes a verification that disorder terms $\Delta E$ in Eq. (2) [56] can be neglected except for the highest dephasing rates. For those rates, dimer atoms were actually accelerated by (a single realization of) the disorder potential, masking the dipole-dipole Zeno effect.

Control through decoherence.-Employing decoherence and dissipation to engineer quantum states is becoming an active field of research $[35,36,44-46,65]$. The preceding example already demonstrates a type of control over the motional state through decoherence. An attractive feature of EIT imaging is the possibility to vary the resulting 


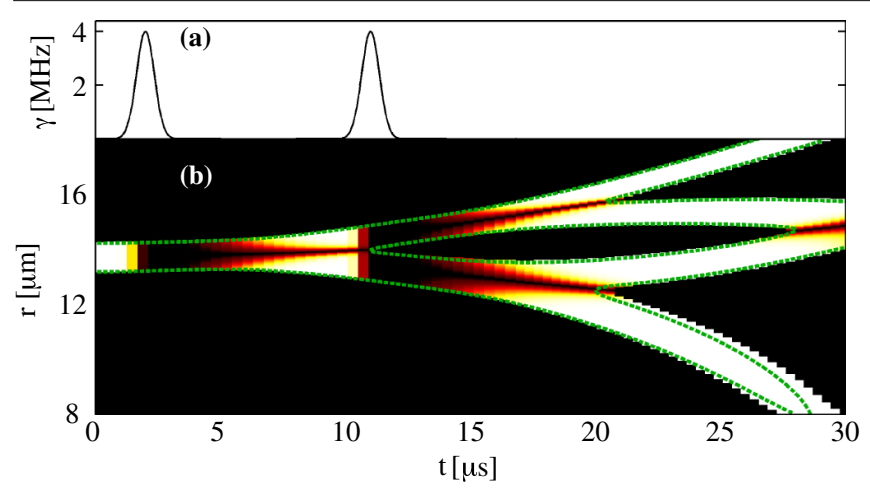

FIG. 4. Repeated application of dephasing pulses with $\gamma_{0}=$ $4 \mathrm{MHz}$ can control relative system populations on the repulsiveand attractive energy surfaces. (a) Time-dependent dephasing rate $\gamma(t)$. (b) Resulting local electronic purity $P_{\text {loc }}(x, t)$; see text. Black to white shades represent the range $[0,1]$. Green dashed lines are contours of the radial probability density $n(r, t)$ at $5 \%$ of its initial peak value.

decoherence rate $\gamma(t)$ in time. Let us consider two Gaussian pulses, $\gamma(t)=\sum_{n=1}^{2} \gamma_{0} \exp \left[-\left(t-t_{n 0}\right)^{2} / \tau^{2}\right]$, at times $t_{n 0}$ with durations $\tau$.

We show in Fig. 4 that strong pulses with $\gamma_{0}=4 \mathrm{MHz}$ can control surface populations by quickly spreading them from one energy surface onto both in an incoherent fashion. The simulation begins in the pure repulsive initial state (3). The decohering pulse around $t_{10}=2 \mu \mathrm{s}$ rapidly turns this into the mixed state $\hat{\rho}_{M}$, which contains both surfaces. Because of dipole-dipole interactions, coherence slowly revives and the populations of the dimer on the repulsive and attractive surfaces subsequently spatially demix, as can be seen from blue lines in panel (b) around $10 \mu \mathrm{s}$. We also show the local electronic purity, defined as $P_{\mathrm{loc}}(r, t)=$ $\left\{\left[\rho(r, r)_{11}^{2}+2 \rho(r, r)_{12} \rho(r, r)_{21}+\rho(r, r)_{22}^{2}\right] /\left[\rho(r, r)_{11}^{2}+\right.\right.$ $\left.\left.\rho(r, r)_{22}^{2}\right]\right\}-1$. We have chosen $P_{\text {loc }}(r, t)$ such that $P=1$ when the electronic state at distance $r\left[\hat{\rho}_{\mathrm{el}}(r)=\langle r|\hat{\rho}| r\rangle\right]$ is pure and $P=0$ when it is mixed. Only after spatial demixing do we find a full revival of the local purity. The dimer components on the repulsive and attractive surface are then decohered a second time around $t_{20}=11 \mu \mathrm{s}$, resulting, again with some delay, in a total of four separate phase-space components. Once these have spatially segregated $(t \approx 23 \mu \mathrm{s})$, the local purity returns to unity, since the system reaches everywhere a pure state.

Practical implementation.-The Rydberg electron experiences direct contact interactions with surrounding ground-state atoms, in addition to the controllable interaction via EIT [66]. This can cause a reduction of Rydberg state lifetimes $[24,26]$ and residual decoherence. Since a rate $\gamma=1 \mathrm{MHz}$ can be realized at relatively low densities $n_{0}=5 \times 10^{17} \mathrm{~m}^{-3}$ [56], with a mean number of only 0.25 ground-state atoms in the Rydberg orbital volume, we expect these effects to be small, with an extrapolated Rydberg dimer lifetime of about $27 \mu$ s. Also (dressed) forces acting on the ground-state atom are small.
Conclusions and outlook.-We have shown how a BO surface can decohere, when constituent electronic basis states are separately coupled to decohering environments. In our example, forces controlling a dimer of Rydberg atoms are gradually turned from repulsive to attractive or may even be entirely suppressed, through decoherence of the superposition of two-atom electronic states that furnish the underlying molecular potential. Our results highlight the importance of intramolecular quantum coherence for the definition of chemical BornOppenheimer surfaces. Similar decoherence effects in chemistry might arise for large bond lengths with atypically dense solvents. Our results also open up a research arena on the influence of tunable decoherence on energy transport and atomic motional dynamics in flexible Rydberg aggregates [14,15,57-59] embedded in host atom clouds $[67,68]$. Additionally, we have shown how temporally localized decoherence pulses can be used as an incoherent means of population redistribution among BO surfaces.

In the present work we consider mainly parameters where the disorder potential $\Delta E$ of the dimer due to its interaction with randomly located background atoms can be neglected. For other choices of parameters the disorder potential can be made dominant over dephasing [21], suggesting an accessible model system for quantum motion in disordered potentials. The combination of decoherence channels discussed here with intersecting BO surfaces $[14,15]$ may allow accessible laboratory model studies of quantum chemical phenomena such as relaxation across a conical intersection [69].

We gladly acknowledge interesting discussions with Alexander Eisfeld, Michael Genkin, Karsten Leonhardt, Jan-Michael Rost, David Schönleber, Varadharajan Srinivasan, and Shannon Whitlock, as well as EU financial support received from the Marie Curie Initial Training Network (ITN) COHERENCE.

*sebastian@iiserb.ac.in

[1] T. F. Gallagher, Rydberg Atoms (Cambridge University Press, Cambridge, England, 1994).

[2] C. H. Greene, A. S. Dickinson, and H. R. Sadeghpour, Phys. Rev. Lett. 85, 2458 (2000).

[3] V. Bendkowsky, B. Butscher, J. Nipper, J. P. Shaffer, R. Löw, and T. Pfau, Nature (London) 458, 1005 (2009).

[4] I. C. H. Liu, J. Stanojevic, and J.-M. Rost, Phys. Rev. Lett. 102, 173001 (2009).

[5] K. R. Overstreet, A. Schwettmann, J. T. ad D Booth, and J. P. Shaffer, Nat. Phys. 5, 581 (2009).

[6] B. Butscher, J. Nipper, J. B. Balewski, L. Kukota, V. Bendkowsky, R. Löw, and T. Pfau, Nat. Phys. 6, 970 (2010).

[7] V. Bendkowsky, B. Butscher, J. Nipper, J. B. Balewski, J. P. Shaffer, R. Löw, T. Pfau, W. Li, J. Stanojevic, T. Pohl et al., Phys. Rev. Lett. 105, 163201 (2010). 
[8] W. Li, T. Pohl, J. M. Rost, S. T. Rittenhouse, H. R. Sadeghpour, J. Nipper, B. Butscher, J. B. Balewski, V. Bendkowsky, R. Loew et al., Science 334, 1110 (2011).

[9] A. Gaj, A. T. Krupp, J. B. Balewski, R. Löw, S. Hofferberth, and T. Pfau, Nat. Commun. 5, 4546 (2014).

[10] A. Gaj, A. T. Krupp, P. Ilzhofer, R. Löw, S. Hofferberth, and T. Pfau, Phys. Rev. Lett. 115, 023001 (2015).

[11] J. Wang, J. N. Byrd, I. Simbotin, and R. Côté, Phys. Rev. Lett. 113, 025302 (2014).

[12] D. Yarkony, J. Phys. Chem. A 105, 6277 (2001).

[13] W. Domcke, D. R. Yarkony, and H. Köppel, Conical Intersections (World Scientific, Singapore, 2004).

[14] S. Wüster, A. Eisfeld, and J. M. Rost, Phys. Rev. Lett. 106, 153002 (2011).

[15] K. Leonhardt, S. Wüster, and J.-M. Rost, Phys. Rev. Lett. 113, 223001 (2014).

[16] K. Leonhardt, S. Wüster, and J. M. Rost, J. Phys. B 50, 054001 (2017).

[17] M. Schlesinger and W. T. Strunz, Phys. Rev. A 77, 012111 (2008).

[18] C. Lienau and A. H. Zewail, J. Phys. Chem. 100, 18629 (1996).

[19] S. Wallentowitz, I. A. Walmsley, L. J. Waxer, and T. Richter, J. Phys. B 35, 1967 (2002).

[20] A. W. Jasper and D. G. Truhlar, J. Chem. Phys. 123, 064103 (2005).

[21] D. W. Schönleber, A. Eisfeld, M. Genkin, S. Whitlock, and S. Wüster, Phys. Rev. Lett. 114, 123005 (2015).

[22] R. Heidemann, U. Raitzsch, V. Bendkowsky, B. Butscher, R. Löw, and T. Pfau, Phys. Rev. Lett. 100, 033601 (2008).

[23] M. Viteau, M. G. Bason, J. Radogostowicz, N. Malossi, D. Ciampini, O. Morsch, and E. Arimondo, Phys. Rev. Lett. 107, 060402 (2011).

[24] J. B. Balewski, A. T. Krupp, A. Gaj, D. Peter, H. P. Büchler, R. Löw, S. Hofferberth, and T. Pfau, Nature (London) 502, 664 (2013).

[25] T. Karpiuk, M. Brewczyk, K. Rzążewski, J. B. Balewski, A. T. Krupp, A. Gaj, R. Löw, S. Hofferberth, and T. Pfau, New J. Phys. 17, 053046 (2015).

[26] T. Niederprüm, O. Thomas, T. Manthey, T. M. Weber, and H. Ott, Phys. Rev. Lett. 115, 013003 (2015).

[27] N. Thaicharoen, A. Schwarzkopf, and G. Raithel, Phys. Rev. A 92, 040701(R) (2015).

[28] N. Thaicharoen, L. F. Gonçalves, and G. Raithel, Phys. Rev. Lett. 116, 213002 (2016).

[29] R. C. Teixeira, C. Hermann-Avigliano, T. L. Nguyen, T. Cantat-Moltrecht, J. M. Raimond, S. Haroche, S. Gleyzes, and M. Brune, Phys. Rev. Lett. 115, 013001 (2015).

[30] R. Faoro, C. Simonelli, M. Archimi, G. Masella, M. M. Valado, E. Arimondo, R. Mannella, D. Ciampini, and O. Morsch, Phys. Rev. A 93, 030701 (2016).

[31] B. Olmos, W. Li, S. Hofferberth, and I. Lesanovsky, Phys. Rev. A 84, 041607(R) (2011).

[32] G. Günter, M. Robert-de-Saint-Vincent, H. Schempp, C. S. Hofmann, S. Whitlock, and M. Weidemüller, Phys. Rev. Lett. 108, 013002 (2012).

[33] G. Günter, H. Schempp, M. Robert-de-Saint-Vincent, V. Gavryusev, S. Helmrich, C. S. Hofmann, S. Whitlock, and M. Weidemüller, Science 342, 954 (2013).
[34] R. Mukherjee, C. Ates, Weibin Li, and S. Wüster, Phys. Rev. Lett. 115, 040401 (2015).

[35] H. Schempp, G. Günter, S. Wüster, M. Weidemüller, and S. Whitlock, Phys. Rev. Lett. 115, 093002 (2015).

[36] D. W. Schönleber, C. D. B. Bentley, and A. Eisfeld, arXiv:1611.02914 [Phys. Rev. Lett. (to be published)].

[37] In writing all states $|\varphi\rangle$ (molecular or Rydberg), we have suppressed electron spin and antisymmetrization under fermion exchange. For Rydberg systems this is a common procedure (and fully justified), since the complete lack of electron overlap between the two atoms due to their large interatomic separation makes symmetrization inconsequential [38].

[38] K. Singer, J. Stanojevic, M. Weidemüller, and R. Côté, J. Phys. B 38, S295 (2005).

[39] M. R. Kutteruf and R. R. Jones, Phys. Rev. Lett. 108, 013001 (2012).

[40] W. R. Anderson, M. P. Robinson, J. D. D. Martin, and T. F. Gallagher, Phys. Rev. A 65, 063404 (2002).

[41] T. Zhou, S. Li, and R. R. Jones, Phys. Rev. A 89, 063413 (2014).

[42] B. Misra and E. C. G. Sudarshan, J. Math. Phys. (N.Y.) 18, 756 (1977).

[43] A. G. Kofman and G. Kurizki, Nature (London) 405, 546 (2000).

[44] M. Tiersch and H. J. Briegel, Phil. Trans. R. Soc. A 370, 4517 (2012).

[45] C. Ates, B. Olmos, Weibin Li, and I. Lesanovsky, Phys. Rev. Lett. 109, 233003 (2012).

[46] M. Lemeshko and H. Weimer, Nat. Commun. 4, 2230 (2013).

[47] M. Fleischhauer, A. Imamoglu, and J. P. Marangos, Rev. Mod. Phys. 77, 633 (2005).

[48] I. Friedler, D. Petrosyan, M. Fleischhauer, and G. Kurizki, Phys. Rev. A 72, 043803 (2005).

[49] A. K. Mohapatra, T. R. Jackson, and C. S. Adams, Phys. Rev. Lett. 98, 113003 (2007).

[50] S. Mauger, J. Millen, and M. P. A. Jones, J. Phys. B 40, F319 (2007).

[51] A. K. Mohapatra, M. G. Bason, B. Butscher, K. J. Weatherill, and C. S. Adams, Nat. Phys. 4, 890 (2008).

[52] H. Schempp, G. Günter, C. S. Hofmann, C. Giese, S. D. Saliba, B. D. DePaola, T. Amthor, M. Weidemüller, S. Sevinçli, and T. Pohl, Phys. Rev. Lett. 104, 173602 (2010).

[53] S. Sevinçli, C. Ates, T. Pohl, H. Schempp, C. S. Hofmann, G. Günter, T. Amthor, M. Weidemüller, J. D. Pritchard, D. Maxwell et al., J. Phys. B 44, 184018 (2011).

[54] V. Parigi, E. Bimbard, J. Stanojevic, A. J. Hilliard, F. Nogrette, R. Tualle-Brouri, A. Ourjoumtsev, and P. Grangier, Phys. Rev. Lett. 109, 233602 (2012).

[55] F. Robicheaux, J. V. Hernández, T. Topçu, and L. D. Noordam, Phys. Rev. A 70, 042703 (2004).

[56] See Supplemental Material at http://link.aps.org/ supplemental/10.1103/PhysRevLett.119.013001 for the derivation of Eq. (2) and the complete Hamiltonian studied in Ref. [21].

[57] C. Ates, A. Eisfeld, and J. M. Rost, New J. Phys. 10, 045030 (2008).

[58] S. Wüster, C. Ates, A. Eisfeld, and J. M. Rost, Phys. Rev. Lett. 105, 053004 (2010). 
[59] S. Möbius, S. Wüster, C. Ates, A. Eisfeld, and J. M. Rost, J. Phys. B 44, 184011 (2011).

[60] G. R. Dennis, J. J. Hope, and M. T. Johnsson, Comput. Phys. Commun. 184, 201 (2013).

[61] G. R. Dennis, J. J. Hope, and M. T. Johnsson, http://www .xmds.org/.

[62] We show an isocontour of $\left[n_{\text {rep }}(t) \rho\left(r, r^{\prime}\right)_{11}^{(0)}(t)\right]$ at one-tenth of its peak value, where $\rho\left(r, r^{\prime}\right)_{11}^{(0)}(t)$ is taken from the corresponding simulation with $\gamma=0$, and $n_{\text {rep }}(t)$ the (diminishing) population on the repulsive surface for $\gamma=0.2 \mathrm{MHz}$.

[63] Explicitly, we can write the off-diagonal element as $\left\langle\pi_{2}\left|\hat{\rho}^{(e l)}\right| \pi_{1}\right\rangle=\int d r \rho(r, r)_{21}$.
[64] We take the final kinetic energy $E_{\text {kin }}=\operatorname{Tr}\left[\hat{\rho}\left(-\hbar^{2} \nabla^{2} / m\right)\right]$; see Ref. [56] at $t=t_{f}$ and subtract the initial zero point energy $\left(E_{\text {kin }}\right.$ at $\left.t=0\right)$.

[65] F. Verstraete, M. M. Wolf, and J. Ignacio Cirac, Nat. Phys. 5, 633 (2009).

[66] C. H. Greene, A. S. Dickinson, and H. R. Sadeghpour, Phys. Rev. Lett. 85, 2458 (2000).

[67] S. Möbius, M. Genkin, S. Wüster, A. Eisfeld, and J.-M. Rost, Phys. Rev. A 88, 012716 (2013).

[68] S. Wüster, S. Möbius, M. Genkin, A. Eisfeld, and J.-M. Rost, Phys. Rev. A 88, 063644 (2013).

[69] S. Perun, A. L. Sobolewski, and W. Domcke, J. Am. Chem. Soc. 127, 6257 (2005). 